\title{
Mathematical modeling of sedimentation of nanoparticles in the vessel of finite depth
}

\author{
S. I. Ezhenkova \\ ITMO University, Kronverkskiy, 49, St. Petersburg, 197101, Russia \\ sveta.ejenkova@yandex.ru
}

DOI 10.17586/2220-8054-2017-8-2-236-238

\begin{abstract}
The sedimentation of nanoparticles in a liquid considering their Brownian diffusion was investigated by using mathematical modeling. The main purpose of this work is investigation of the particles' behavior in the area adjoining to the bottom of the vessel - the boundary layer.
\end{abstract}

Keywords: sedimentation, diffusion, the Newton polygon.

Received: 15 January 2017

Revised: 8 March 2017

\section{Introduction}

The problem of the influence of Brownian diffusion of nanoparticles on their sedimentation remains relevant [1]. Since the experiment of Robert Brown (the movement of pollen particles in a liquid drop), many scientists have studied the behavior of particles in different types of environments. The method of nanoparticle synthesis plays an important role in the distribution of nanoparticles' sizes [2]. Theoretical investigation of the sedimentation of nanoparticles is described in [3,4], Brownian motion of nanoparticles is described in [5-7].

In this work, we discuss the effect of the nanoparticles' diffusion on their sedimentation along the lower boundary of the vessel bottom, called the boundary layer [8]. To find the solution of the diffusion equation, the Newton polygon method is used - a method allowing one to find the solution of the equation with perturbed coefficients.

\section{The calculation of particles density distribution in the boundary layer}

We consider the sedimentation process of spherical nanoparticles in a liquid taking into account the Brownian diffusion between them. The equation of convective diffusion has the form:

$$
\frac{\partial f}{\partial t}+v(R) \frac{\partial f}{\partial x}=D(R) \frac{\partial^{2} f}{\partial x^{2}}
$$

where $v(R)$ is the velocity of sedimentation, $f$ is the particle distribution function, $D$ is the diffusion coefficient, $x$ is the coordinate, $t$ is the time of sedimentation.

The initial condition:

$$
\left.f\right|_{t=0}=f_{0}(R) \Theta(x),
$$

where $f_{0}(R)$ is the size distribution function, $\Theta(x)$ is the coordinate distribution function (the Heaviside step function).

The boundary conditions:

$$
\begin{aligned}
& \left.j\right|_{x=0}=0, \\
& \left.j\right|_{x=L}=0,
\end{aligned}
$$

where $j=v(R) f-D \frac{d f}{d x}$ is the particles' flux density, $L$ is the height of the vessel.

To find the solution inside the boundary layer, we transform (1) into dimensionless form. For this, we introduce the dimensionless parameters for variables $x, t$ and some small parameter $\varepsilon$ :

$$
x=L \bar{x}, \quad t=T \bar{t}, \quad \varepsilon=\frac{D(R)}{v(R) L},
$$

where $T=\frac{L}{v(R)}, v(R)=\gamma R^{2}=\frac{2}{9} \frac{g\left(\rho-\rho_{p}\right)}{\mu} R^{2}$ is the velocity of sedimentation, $\rho, \rho_{p}$ is the liquid density and particle density, $\mu$ is the viscosity, $D=\frac{k_{B} T}{6 \pi \mu R}$ is the diffusion coefficient, where $k_{B}$ is the Boltzmann constant, $T$ is the temperature, $R$ is the radius of particle, $g$ is the gravitational constant. 
Here, the value of $\varepsilon$ is a small considering the fact that the diffusion of the particles is small: the larger the radius of the particle, the lower its diffusion.

Then, we rewrite the equation in the new form:

$$
\varepsilon \frac{\partial^{2} f}{\partial \bar{x}^{2}}-\frac{\partial f}{\partial \bar{x}}-\frac{\partial f}{\partial \bar{t}}=0
$$

and introduce a new coordinate $\xi=e^{-\lambda}(1-\bar{x})$, where $\lambda$ is the indefinite parameter.

After all this transformation, (1) takes the form:

$$
\varepsilon^{1-2 \lambda} \frac{\partial^{2} f}{\partial \xi^{2}}+\varepsilon^{-\lambda} \frac{\partial f}{\partial \xi}-\varepsilon^{0} \frac{\partial f}{\partial \bar{t}}=0 .
$$

For sewing the solution $f^{p}(\xi)$ inside the boundary layer with the solution $f^{0}(\bar{x}, \bar{t})$ outside the boundary layer, the following asymptotic equality must hold:

$$
\lim _{\bar{x} \rightarrow 1} f^{0}(\bar{x}, \bar{t})=\lim _{\xi \rightarrow \infty} f^{p}(\xi)
$$

Firstly, we consider the behavior of individual members of (3) taking into account $\varepsilon \rightarrow 0$ for different values of $\lambda$. For this, we use the Newton polygon.

(1) When $0<\lambda<1$, the leading term of the equation has the degree $-\lambda$. In this case, the solution of (3) has only one arbitrary constant and satisfies only one of two boundary conditions (2);

(2) When $\lambda>1$ the leading term of the equation has the degree $1-2 \lambda$. In this case, the solution of (3) has two arbitrary constants and is satisfied by two boundary conditions (2), but in this case $\lim _{\xi \rightarrow \infty} f^{p}(\xi)$, does not exist for this solution. Consequently, this solution cannot be sewed with the solution outside the boundary layer;

(3) When $\lambda=1$ there are two leading terms with the degrees $1-2 \lambda$ and $-\lambda$ which satisfy (2).

After this, we find the solution of (3) outside the boundary layer:

$$
f^{0}(x, t)=A R e^{-\lambda R^{2}} \Theta(\bar{x}-\bar{t})
$$

and the solution of (3) inside the boundary layer:

$$
f^{p}(\xi)=A+B \mathrm{e}^{-\xi}
$$

where $A=f_{0}(R) \Theta(1-\bar{t}), B=\frac{f_{0}(R) \Theta(1-\bar{t})}{\varepsilon^{-1}+1}, \lambda=1$, and carry out the sewing of this solutions in accordance with (4).

In the result, we have the final formula for the particle size distribution:

$$
f=f_{0}(R) \Theta\left(1-\frac{t v(R)}{L}\right)\left(1+\frac{D(R)}{D(R)+v(R) L} e^{-\frac{v(R)}{D(R)}(L-x)}\right) .
$$

This equation allows us to see influence of diffusion localization along the lower boundary at the bottom of the vessel. The exponential dependence of the concentration of particles on the coordinate arises in the localization region, while in an area remote from the bottom, conventional particle sedimentation occurs. The order of the width of the boundary layer is $\frac{D(R)}{v(R)}$ for different particle sizes. Sedimentation velocity and diffusion are the functions of the particles radius. In this regard, if the thickness of the boundary layer is visually observed (optically, for example), then it makes it possible to estimate the particle sizes without taking measurements.

\section{Acknowledgements}

This work was partially supported financially by grant 074-U01 of the Government of the Russian Federation, by grant MK-5161.2016.1 of the President of the Russian Federation, by grant 16-11-10330 of Russian Science Foundation. 


\section{References}

[1] Vahedi Tafreshi H., Piseri P., Barborini E., Benedek G. and Milani P. Simulation on the effect of Brownian motion on nanoparticle trajectories in a pulsed microplasma cluster source. Journal of Nanoparticle Research, 2002, 4(6), P. 511-524.

[2] Almjasheva O.V. Formation and structural transformations of nanoparticles in the $\mathrm{TiO}_{2}-\mathrm{H}_{2} \mathrm{O}$ system. Nanosystems: Physics, Chemistry, Mathematics, 2016, 7(6), P. 1031-1049.

[3] Gangulya S., Chakrabortyb S. Sedimentation of nanoparticles in nanoscale colloidal suspensions. Physics Letters A, 2011, 375, P. 23942399.

[4] Demeler B., Tich-Lam Nguyen, Gorbet G.E., Schirf V., Brookes E.H., Mulvaney P., El-Ballouli A.O., Pan J., Bakr O.M., Demeler A.K., Hernandez Uribe B.I., Bhattarai N., Whetten R.L. Characterization of size, anisotropy and density heterogeneity of nanoparticles by sedimentation velocity. Analytical Chemistry, 2014, 86, P. 7688-7695.

[5] Eun Chul Cho, Qiang Zhang, Younan Xia. The effect of sedimentation and diffusion on cellular uptake of gold nanoparticles. Nature Nanotechnology, 2011, 6, P. 385-391.

[6] Ezhenkova S.I., Chivilikhin S.A. Mathematical modeling of sedimentation process of nanoparticles in gradient medium. Journal of Physics: Conference Series, 2015, 643, P. 012111.1-012111.6.

[7] Fisenko S.P., Shnip A.I. Physics, chemistry and applications of nanoctructures. World Scientific, Singapore, 2003,596 pp.

[8] Nayfeh A.H. Perturbation Methods. Wiley-Interscience, USA, 1973, 425 pp. 